\title{
Designing bivariate timeseries with controlled Granger causality
}

\author{
Shohei Hidaka (JAIST) Takuma Torii (JAIST)
}

January 29, 2021

\begin{abstract}
In this manuscript, we analyzed vector auto-regressive (VAR) model in order to draw a design principle for bivariate timeseries with a controlled statistical inter-relationship. We show how to generate bivariate timeseries with given covariance and Granger causality, and showed the trade-off relationship between these two types of statistical interaction. In principle, covariance and Granger causality are controllable independently, but the ranges of their values, which let VAR be proper and have stationary distribution, are constrained by each other. Thus, there is an essential tri-lemma among the stability and properness of VAR, the controllability of covariance, and that of Granger causality.
\end{abstract}

\section{Background and motivation}

In a field of cognitive psychology, human perception of life-likeness, called animacy perception, of one or multiple moving geometric patterns has been studied for decades $[5,2,3,8,6]$. There are multiple findings on the effect of "synchrony" or "temporal contingency" between the multiple moving points on animacy perception. Findings from one line of research [2] have suggested that higher degree of "temporal contingency" of the moving objects is related to higher likelihood of animacy perception. Findings from the other line of research [7] have suggested that the highest "temporal contingency", presented in a form of perfect synchronization, would decrease the likelihood of animacy perception.

These two lines of research together have suggested the existence of multiple types of "temporal contingency", which may not be yet clarified in these past research, and two lines of apparent conflicting effects of "temporal contingency" may be caused by confusion of these two distinct types of effects. 
With this potential conflict in the literature on animacy perception in our mind, we explore a theoretical framework, which can generate timeseries of multiple random variables with multiple distinct types of statistical dependency. One of such systems, which is simple enough and readily manipulable, is vector auto-regression (VAR). Vector auto-regression is a random process generating multivariate timeseries for a given set of parameters. In this manuscript, we specifically consider only bivariate VAR, which is a minimal system with interaction between two moving points.

Importantly, bivariate VAR, a series of paired random variables $\left(x_{t}, y_{t}\right)$ for $t=0,1, \ldots$, has two types of statistical dependency - correlation and Granger causality of timeseries [4]. Correlation between univariate series $x$ and $y$ is statistical dependency between $x_{t}$ and $y_{t}$ in the limit $t \rightarrow \infty$ (if exists), while (one side of) Granger causality from $y$ to $x$ is that between $x_{t}$ and $y_{t-1}$ given $x_{t-1}$ in the limit $t \rightarrow \infty$ (if exists). Conceptually, correlation captures a type of similarity between two timeseries, meanwhile, Granger causality captures "reactiveness" of one timeseries to the other.

Thus, given these difference in theory, we hypothesize that these two theoretically distinct statistical dependency may clarify the potential multiple sources of temporal contingency being involved with human animacy perception of multiple moving points. In order to test this hypothesis, we need to design a bivariate timeseries generated by VAR with controlled their variance, correlation, and two ways of Granger causality. In this manusrcipt, thus, we explore a theoretical structure of bivariate VAR from the designer's perspective, and analyzed mathematical limit to which we can control a bivariate timeseries with a certain statistical dependency.

This manuscript is written as the following structure. In Section 2, VAR model is defined, and derive a set of basic statistical properties of VAR model such as Granger causality (Section 3) with a set of parameters. In Section 4, the existence of stationary distribution of VAR is analyzed. This is a foundation which gives the limit of controllable set of parameters. In Section 5, we give a method to derive the parameters of VAR for a given set of statistics in bivariate timeseries. In Section 6, the mathematical analysis provided in this manuscript is summarized and add a remark on a design principle of bivariate timeseries generated by VAR.

\section{Vector Auto-Regression (VAR)}

In theory, Granger causality (GC) is transfer entropy of the random variables in the bivariate vector auto-regression (VAR) model up to the constant 
factor of 2, if the VAR model has a stationary distribution [1]. Thus, it is straight-forward to start with the bivariate VAR and derive its transfer entropy. In this way, we can derive a rich mathematical relationship between GC and the properties of VAR, no just as a statistics of bivariate timeseries.

Definition 1. For some real vector $\mu \in \mathbb{R}^{2}$ and some positive definite matrix $\Sigma \in \mathbb{R}^{2 \times 2}$, suppose the random variable $\epsilon_{t}$ for every integer $t=0,1, \ldots$ is drawn from the bivariate normal distribution $N\left(\epsilon_{t} \mid \mu, \Sigma\right)$ with mean $\mu \mathbb{R}^{2}$ and variance $\Sigma$. Define the initial vector by $v_{0}=\left(\begin{array}{l}x_{0} \\ y_{0}\end{array}\right)$, and fort $\geq 0$ and a given matrix with real entries $A=\left(\begin{array}{cc}a_{0,0} & a_{0,1} \\ a_{1,0} & a_{1,1}\end{array}\right) \in \mathbb{R}^{2 \times 2}$, define the random variable $v_{t}$ by

$$
v_{t+1}:=A v_{t}+\epsilon_{t}
$$

Then, bivariate vector auto-regression is defined by the semi-infinite series of random variables $V=\left(v_{0}, v_{1}, v_{2}, \ldots\right)$.

\subsection{Marginal distribution of the VAR at each step}

Lemma 1 (Marginal distribution of the VAR random variables at each step). The VAR model with the initial vector $v_{0} \in \mathbb{R}^{2}$ and the coefficient matrix $A \in \mathbb{R}^{2 \times 2}$ has the bivariate normal distribution

$$
N\left(v_{t} \mid \mu_{t}, \Sigma_{t}\right)
$$

as its marginal distribution of the random variable $v_{t}$ at each step $t=$ $0,1, \ldots$, where

$$
\mu_{t}:=A^{t} v_{0}, \quad \Sigma_{t}=\sum_{s=0}^{t} A^{s} \Sigma A^{\top s} .
$$

Proof. By Definition 1, Lemma 1 holds for $t=0$. For $t+1>0$, we prove Lemma 1 by assuming that it holds up to $t \geq 0$. By this assumption held for $t$, we have the distribution of $v_{t} \in \mathbb{R}^{2}$ as the bivariate normal distribution

$$
N\left(v_{t} \mid \mu_{t}, \Sigma_{t}\right)=(2 \pi)^{-1}\left|\Sigma_{t}\right|^{-\frac{1}{2}} e^{-\frac{1}{2}\left(v_{t}-\mu_{t}\right)^{\top} \Sigma_{t}^{-1}\left(v_{t}-\mu_{t}\right)}
$$

with its mean $\mu_{t}$, and its covariance matrix $\Sigma_{t}$. Then the random variable $A v_{t}$ is distributed by the normal distribution

$$
\begin{aligned}
N\left(A v_{t} \mid A \mu_{t}, A \Sigma_{t} A^{\top}\right) & =(2 \pi)^{-1}\left|A \Sigma_{t} A^{\top}\right|^{-\frac{1}{2}} \\
& \times e^{-\frac{1}{2}\left(A\left(v_{t}-\mu_{t}\right)\right)^{\top}\left(A \Sigma_{t} A^{\top}\right)^{-1}\left(A\left(v_{t}-\mu_{t}\right)\right)}
\end{aligned}
$$


with its mean $A \mu_{t}$, and the covariance matrix $A \Sigma_{t} A^{\top}$. The random variable $\epsilon_{t}$ is distributed by the following normal distribution

$$
N\left(\epsilon_{t} \mid 0, \Sigma\right)=(2 \pi)^{-1}|\Sigma|^{-\frac{1}{2}} e^{-\frac{1}{2} \epsilon_{t}^{\top} \Sigma^{-1} \epsilon_{t}} .
$$

Thus, by VAR Eq (1), we have the random variable

$$
v_{t+1}:=A v_{t}+\epsilon_{t}
$$

has the distribution calculated by the following integral:

$$
P\left(v_{t+1}\right)=\int_{\epsilon_{t} \in \mathbb{R}^{2}} N\left(v_{t+1}-\epsilon_{t} \mid A \mu_{t}, A \Sigma_{t} A^{\top}\right) N\left(\epsilon_{t} \mid 0, \Sigma\right) \mathrm{d} \epsilon_{t} .
$$

Calculating this, we have

$$
P\left(v_{t+1}\right)=N\left(v_{t+1} \mid A \mu_{t}, A \Sigma_{t} A^{\top}+\Sigma\right) .
$$

Thus defining by $\mu_{t+1}:=A \mu_{t}$ and $\Sigma_{t+1}=A \Sigma_{t} A^{\top}+\Sigma$, Lemma 1 holds for $t+1$. By expanding this, we have the Lemma 1 for any integer $t \geq 0$.

\subsection{Lyapunov equation}

By Lemma 1

$$
\mu_{t}=A^{t} \text { and } \Sigma_{t}=\sum_{s=0}^{t} A^{s} \Sigma\left(A^{s}\right)^{\top} .
$$

From this, we have stationary distribution

$$
\lim _{t \rightarrow \infty} N\left(v_{t} \mid \mu_{t}, \Sigma_{t}\right)
$$

if and only if the absolute values of all the eigenvalues $\lambda_{0}, \lambda_{1}$ of the coefficient matrix $A$ are less than 1 . If there is such stationary distribution, we call VAR is stable, and its stationary distribution is the bivariate normal distribution

$$
N\left(\hat{v} \mid \mathbf{0}_{2}, \hat{\Sigma}\right) .
$$

Thus, if the VAR is stable, we have the following Lyapunov equation of the stationary covariance matrix $\hat{\Sigma} \in \mathbb{R}^{2 \times 2}$ :

$$
\hat{\Sigma}=\Sigma+A \hat{\Sigma} A^{\top} .
$$

The Lyapunov equation is solved analytically by

$$
\operatorname{vec}(\hat{\Sigma})=\left(I_{4}-A \otimes A\right)^{-1} \operatorname{vec}(\Sigma),
$$


where $\otimes$ denotes the Kronecker product, and $\operatorname{vec}(X)$ for any matrix $X=$ $\left(x_{i, j}\right)_{i=1, \ldots, n, j=1, \ldots, m}$ is the vectorization operator $\operatorname{vec}(): \mathbb{R}^{n \times m} \rightarrow \mathbb{R}^{n m \times 1}$ defined by

$$
\operatorname{vec}(X):=\left(x_{1,1}, x_{2,1}, \ldots, x_{n, 1}, \ldots, x_{1, m}, x_{2, m}, \ldots, x_{n, m}\right)^{\top} .
$$

The Lyapunov equation 4 has the solution for $\hat{\Sigma}$, if the VAR is stable. This is shown by Lemma 4 .

\section{Transfer entropy and Granger causality}

In [1], transfer entropy of an appropriate triplet of variables in VAR model is shown to be equivalent to Granger causality up to the constant factor 2 . Following this guid, we defined this quantity as Granger causality of VAR model as follows.

Although this relationship has been known in a more general form [1], we re-derive it for bivariate VAR in order to analyze the structure of VAR and GCs in depth, such as its upper and lower bound (Lemma 3), stability (Section 4), and design principle (Section 5), later on.

Definition 2. If VAR with its random variables $v_{t}=\left(x_{t}, y_{t}\right)^{\top} \in \mathbb{R}^{2}$ is stable, transfer entropy from $y$ to $x$ is defined by

$$
T_{y \rightarrow x}:=\lim _{t \rightarrow \infty}\left(H\left(x_{t+1} \mid x_{t}\right)-H\left(x_{t+1} \mid x_{t}, y_{t}\right)\right),
$$

and the transfer entropy from $x$ to $y$ is defined by

$$
T_{x \rightarrow y}:=\lim _{t \rightarrow \infty}\left(H\left(y_{t+1} \mid y_{t}\right)-H\left(y_{t+1} \mid y_{t}, x_{t}\right)\right),
$$

where the differential entropy of random variable $x$ with its probability density function $P$ is

$$
H(x):=-\int_{x \in \Omega} P(x) \log P(x) \mathrm{d} x,
$$

and the conditional entropy is

$$
H(x \mid y):=H(x, y)-H(y)
$$

In particular, we call two times of transfer entropy Granger causality denoted by

$$
G_{0}=2 T_{y \rightarrow x} G_{1}=2 T_{x \rightarrow y}
$$


Specifically, GCs are given by the following lemma.

Lemma 2 (Granger causality). If a stable VAR has its covariance matrix, coefficient matrix, and stationary matrix

$$
\Sigma=\left(\begin{array}{ll}
\sigma_{0,0} & \sigma_{0,1} \\
\sigma_{1,0} & \sigma_{1,1}
\end{array}\right), A=\left(\begin{array}{ll}
a_{0,0} & a_{0,1} \\
a_{1,0} & a_{1,1}
\end{array}\right), \hat{\Sigma}=\left(\begin{array}{ll}
\hat{\sigma}_{0,0} & \hat{\sigma}_{0,1} \\
\hat{\sigma}_{1,0} & \hat{\sigma}_{1,1}
\end{array}\right)
$$

each Granger causality of this $V A R$ for $i=0,1$ is

$$
G_{i}=\log \left(1+\frac{a_{i, 1-i}^{2} \operatorname{det}(\hat{\Sigma})}{\hat{\sigma}_{i, i} \sigma_{i, i}}\right) .
$$

Proof. In general, the differnetial entropy of multivariate normal disribution $N(v \mid \mu, \Sigma)$ is

$$
H(v)=\frac{1}{2} \log |2 \pi e \Sigma|,
$$

where $e \approx 2.71$ is Napier's constant. For the joint probability distribution of $v_{t}=\left(x_{t}, y_{t}\right)^{\top}$

$$
P\left(v_{t+1} \mid v_{t}\right)=N\left(v_{t+1} \mid A v_{t}, \Sigma\right),
$$

the two marginal probability distributions of $x_{t}, y_{t}$ are

$P\left(x_{t+1} \mid v_{t}\right)=N\left(x_{t+1} \mid(1,0) A v_{t}, \sigma_{0,0}\right)$ and $P\left(y_{t+1} \mid v_{t}\right)=N\left(y_{t+1} \mid(0,1) A v_{t}, \sigma_{1,1}\right)$.

Thus, the conditional entropy of $x_{t+1}$ and $y_{t+1}$ given $v_{t}=\left(x_{t}, y_{t}\right)^{\top}$ are

$$
H\left(x_{t+1} \mid x_{t}, y_{t}\right)=\frac{1}{2} \log \left|2 \pi e \sigma_{0,0}\right| \text { and } H\left(y_{t+1} \mid x_{t}, y_{t}\right)=\frac{1}{2} \log \left|2 \pi e \sigma_{1,1}\right| .
$$

With the conditional probability distribution

$$
P\left(v_{t+1} \mid v_{t}\right)=N\left(v_{t+1} \mid A v_{t}, \Sigma\right)
$$

and the marginal probability distribution

$$
P\left(v_{t}\right)=N\left(v_{t} \mid A^{t} v_{0}, \Sigma_{t}\right),
$$

the joint probability distribution of $v_{t}$ and $v_{t+1}$ is their product

$$
P\left(v_{t+1}, v_{t}\right)=N\left(v_{t+1} \mid A v_{t}, \Sigma\right) N\left(v_{t} \mid A^{t} v_{0}, \Sigma_{t}\right) .
$$

Specifically, this quad-variate normal distribution is $P\left(v_{t+1}, v_{t}\right)=e^{-\frac{1}{2}\left(v_{t+1}-A v_{t}\right)^{\top} \Sigma^{-1}\left(v_{t+1}-A v_{t}\right)-\frac{1}{2}\left(v_{t}-A^{t} v_{0}\right)^{\top} \Sigma_{t}^{-1}\left(v_{t}-A^{t} v_{0}\right)}(2 \pi)^{-2}|\Sigma|^{-\frac{1}{2}}\left|\Sigma_{t}\right|^{-\frac{1}{2}}$. 
Applying the identities

$$
v_{t+1}-A v_{t}=v_{t+1}-A^{t+1} v_{0}-A\left(v_{t}-A^{t} v_{0}\right),
$$

and

$$
\Sigma_{t}^{\prime}:=\left(\begin{array}{cc}
\Sigma+A \Sigma_{t} A^{\top} & A \Sigma_{t} \\
\Sigma_{t} A^{\top} & \Sigma_{t}
\end{array}\right)=\left(\begin{array}{cc}
\Sigma^{-1} & -\Sigma^{-1} A \\
-A^{\top} \Sigma^{-1} & \Sigma_{t}^{-1}+A \Sigma^{-1} A^{\top}
\end{array}\right)^{-1}
$$

and $|\Sigma|\left|\Sigma_{t}\right|=\left|\Sigma_{t}^{\prime}\right|$ to $P\left(v_{t+1}, v_{t}\right)$, we have

$$
P\left(v_{t+1}, v_{t}\right)=N\left(v_{t}^{\prime} \mid \mu_{t}^{\prime}, \Sigma_{t}^{\prime}\right),
$$

where

$$
v_{t}^{\prime}:=\left(\begin{array}{c}
v_{t+1} \\
v_{t}
\end{array}\right), \mu_{t}^{\prime}:=\left(\begin{array}{c}
A^{t+1} v_{0} \\
A^{t} v_{0}
\end{array}\right), \Sigma_{t}^{\prime}:=\left(\begin{array}{cc}
\Sigma+A \Sigma_{t} A^{\top} & A \Sigma_{t} \\
\Sigma_{t} A^{\top} & \Sigma_{t}
\end{array}\right) .
$$

From this joint probability distribution $P\left(v_{t+1}, v_{t}\right)$, we drive the marginal distributions

$$
P\left(x_{t+1}, x_{t}\right)=N\left(x_{t+1}, x_{t} \mid \mu_{t, 0}, \Sigma_{t, 0}\right), P\left(y_{t+1}, y_{t}\right)=N\left(y_{t+1}, y_{t} \mid \mu_{t, 1}, \Sigma_{t, 1}\right) .
$$

where for $i=0,1$ the mean vectors and covariance matrices are defined as follows:

$$
\begin{aligned}
\mu_{t, i}:= & \left(\begin{array}{c}
e_{i}^{\top} A^{t+1} v_{0} \\
e_{i}^{\top} A^{t} v_{0}
\end{array}\right)=\left(I_{2} \otimes e_{i}\right) \mu_{t}^{\prime}, \\
\Sigma_{t, i} & :=\left(\begin{array}{cc}
e_{i}^{\top}\left(\Sigma+A \Sigma_{t} A^{\top}\right) e_{i} & e_{i}^{\top} A \Sigma_{t} e_{i} \\
e_{i}^{\top} \Sigma_{t} A^{\top} e_{i} & e_{i}^{\top} \Sigma_{t} e_{i}
\end{array}\right),=\left(I_{2} \otimes e_{i}\right)^{\top} \Sigma_{t}^{\prime}\left(I_{2} \otimes e_{i}\right),
\end{aligned}
$$

with the unit vectors $e_{0}:=(1,0)^{\top}, e_{1}:=(0,1)^{\top}$.

Thus, we have the joint entropy of $x_{t}$ and $x_{t+1}$

$$
\begin{aligned}
H\left(x_{t+1}, x_{t}\right) & =\frac{1}{2} \log \left|2 \pi e \Sigma_{t, 0}\right| \\
& =\frac{1}{2} \log (2 \pi e)^{2}\left|e_{0}^{\top}\left(\Sigma+A \Sigma_{t} A^{\top}\right) e_{0} e_{0}^{\top} \Sigma_{t} e_{0}-\left(e_{0}^{\top} A \Sigma_{t} e_{0}\right)^{2}\right|
\end{aligned}
$$

and the marginal distribution of $x_{t}$

$$
H\left(x_{t}\right)=\frac{1}{2} \log 2 \pi e\left|e_{0}^{\top} \Sigma_{t} e_{0}\right| .
$$


Using these, we have the conditional entropy

$$
H\left(x_{t+1} \mid x_{t}\right)=\frac{1}{2} \log 2 \pi \frac{e_{0}^{\top}\left(\Sigma+A \Sigma_{t} A^{\top}\right) e_{0} e_{0}^{\top} \Sigma_{t} e_{0}-\left(e_{0}^{\top} A \Sigma_{t} e_{0}\right)^{2}}{\left|e_{0}^{\top} \Sigma_{t} e_{0}\right|} .
$$

By stability of VAR, the Lyapunov equation (4) holds, and this conditional entropy in the limit $t \rightarrow \infty$ is

$$
\lim _{t \rightarrow \infty} H\left(x_{t+1} \mid x_{t}\right)=\frac{1}{2} \log 2 \pi \frac{\left(e_{0}^{\top} \hat{\Sigma} e_{0}\right)^{2}-\left(e_{0}^{\top} A \hat{\Sigma} e_{0}\right)^{2}}{\left|e_{0}^{\top} \hat{\Sigma} e_{0}\right|}
$$

Applying Definition 2 and denoting by entries in the stationary covariance $\operatorname{matrix} \hat{\Sigma}=\left(\begin{array}{cc}\hat{\sigma}_{0,0} & \hat{\sigma}_{0,1} \\ \hat{\sigma}_{1,0} & \hat{\sigma}_{1,1}\end{array}\right)$, we have

$$
G_{0}=2 T_{y \rightarrow x}=\log \frac{\left(\hat{\sigma}_{0,0}\right)^{2}-\left(a_{0,0} \hat{\sigma}_{0,0}+a_{0,1} \hat{\sigma}_{1,0}\right)^{2}}{\hat{\sigma}_{0,0} \sigma_{0,0}}
$$

Similarly, we have

$$
G_{1}=2 T_{x \rightarrow y}=\log \frac{\left(\hat{\sigma}_{1,1}\right)^{2}-\left(a_{1,0} \hat{\sigma}_{0,1}+a_{1,1} \hat{\sigma}_{1,1}\right)^{2}}{\hat{\sigma}_{1,1} \sigma_{1,1}} .
$$

Let us define for $i=0,1$

$$
\delta_{i}:=\left(\hat{\sigma}_{i, i}\right)^{2}-\left(a_{i, i} \hat{\sigma}_{i, i}+a_{i, 1-i} \hat{\sigma}_{1-i, i}\right)^{2} \text { and } \delta_{i}^{\prime}:=\hat{\sigma}_{i, i} \sigma_{i, i} .
$$

By Lyapunov equation, $\sigma_{i, i}=\hat{\sigma}_{i, i}-e_{i}^{\top} A \hat{\Sigma} A^{\top} e_{i}$. Applying this to $\delta_{i}^{\prime}$, we have

$$
\begin{gathered}
\delta_{i}=\hat{\sigma}_{i, i}^{2}-e_{i}^{\top} A\left(\begin{array}{cc}
\hat{\sigma}_{i, i}^{2} & \hat{\sigma}_{i, i} \hat{\sigma}_{1-i, i} \\
\hat{\sigma}_{i, i} \hat{\sigma}_{1-i, i} & \hat{\sigma}_{1-i, i}^{2}
\end{array}\right) A^{\top} e_{i} \\
\delta_{i}^{\prime}=\hat{\sigma}_{i, i}^{2}-e_{i}^{\top} A\left(\begin{array}{cc}
\hat{\sigma}_{i, i} \hat{\sigma}_{0,0} & \hat{\sigma}_{i, i} \hat{\sigma}_{0,1} \\
\hat{\sigma}_{i, i} \hat{\sigma}_{1,0} & \hat{\sigma}_{i, i} \hat{\sigma}_{1,1}
\end{array}\right) A^{\top} e_{i}
\end{gathered}
$$

As $\delta_{i}-\delta_{i}^{\prime}=a_{i, 1-i}^{2} \operatorname{det}(\hat{\Sigma})$ and $G_{0}=\log \left(1+\frac{\delta_{i}-\delta_{i}^{\prime}}{\delta_{i}^{\prime}}\right)$, we

$$
G_{i}=\log \left(1+\frac{a_{i, 1-i}^{2} \operatorname{det}(\hat{\Sigma})}{\hat{\sigma}_{i, i} \sigma_{i, i}}\right)
$$


Lemma 3 (The upper and lower bound for Granger causality). For each $i=0,1$, Granger causality $G_{i}$ has the following bounds:

$$
0 \leq G_{i} \leq \log \gamma_{i}
$$

where $\gamma_{i}:=\frac{\hat{\sigma}_{i, i}}{\sigma_{i, i}} \geq 1$ due to the Lyapunov equation (4). The lower bound $G_{i}=0$ is given only if

$$
a_{i, 1-i}^{2} \operatorname{det}(\hat{\Sigma})=0 .
$$

The upper bound $G_{i}=\log \gamma_{i}$ is given only if

$$
a_{i, i} \hat{\sigma}_{i, i}+a_{i, 1-i} \hat{\sigma}_{1-i, i}=0 .
$$

Proof. As the stationary covariance matrix is (semi-)positive definite, $\operatorname{det}(\hat{\Sigma}) \geq$ 0 . Thus the lower bound of Granger causality is $G_{i} \geq \log (1)=0$ and this bound is only reacheable when $a_{i, 1-i}^{2} \operatorname{det}(\hat{\Sigma})=0$.

Modifying (13) and (14), for $i=0,1$ we have

$$
\left(a_{i, i} \hat{\sigma}_{i, i}+a_{i, 1-i} \hat{\sigma}_{1-i, i}\right)^{2}=\hat{\sigma}_{i, i}\left(\hat{\sigma}_{i, i}-\sigma_{i, i} e^{G_{i}}\right) .
$$

As $\left(a_{i, i} \hat{\sigma}_{i, i}+a_{i, 1-i} \hat{\sigma}_{1-i, i}\right)^{2} \geq 0$ and $\hat{\sigma}_{i, i}>0$,

$$
G_{i} \leq \log \gamma_{i}
$$

This lower bound holds, only if $a_{i, i} \hat{\sigma}_{i, i}+a_{i, 1-i} \hat{\sigma}_{1-i, i}=0$.

\section{Stability and constraints of VAR}

In this study, we primarily consider the class of stable VAR model with a proper set of the parameters. In this class, statistical nature of any VAR is characterized with the base covariance matrix $\Sigma \in \mathbb{R}^{2 \times 2}$, coefficient matrix $A \in \mathbb{R}^{2 \times 2}$, and stationary covariance matrix $\hat{\Sigma} \in \mathbb{R}^{2 \times 2}$. Let us denote the set of (strictly) positive definite matrices by

$$
\mathbb{R}_{+}^{2 \times 2}:=\left\{M \in \mathbb{R}^{2 \times 2} \mid \operatorname{det}(M)>0 \text { and } \operatorname{tr}(M)>0\right\},
$$

and the set of coefficient matrices of stable VAR models

$$
\mathbb{R}_{*}^{2 \times 2}:=\left\{M \in \mathbb{R}^{2 \times 2}|-1+| \operatorname{tr}(M) \mid<\operatorname{det}(M)<1\right\} .
$$

We will shortly show the stable set $\mathbb{R}_{*}^{2 \times 2}$ include all and only coefficient matrices of stable VAR models.

With this notation of the set of matrices, the two conditions any proper VAR model needs to satisfy are as follows. 
Stability Any stable VAR model has both of the eigenvalues $\lambda_{0}, \lambda_{1}$ of its coefficient matrix $A$ be $\left|\lambda_{0}\right|,\left|\lambda_{1}\right|<1$.

Properness To have a proper (non-degenerated) bivariate normal distribution in a VAR model, its base covariance matrix $\Sigma$ and stationary covariance matrix $\hat{\Sigma}$ needs to satisfy $\Sigma, \hat{\Sigma} \in \mathbb{R}_{+}^{2 \times 2}$. The set of positive definite matrices is equivalently written with the entries of matrix

$$
\mathbb{R}_{+}^{2 \times 2}=\left\{C \in \mathbb{R}^{2 \times 2} \mid C_{0,0}>0, C_{1,1}>0, \text { and } C_{0,0} C_{1,1}-C_{0,1} C_{1,0}>0\right\} .
$$

\subsection{Stability of VAR}

As stated previously in Section 2.2, the stability of VAR is primarily characterized by the eigenvalues of the coefficient matrix $A$. But this condition is equivalent with $A \in \mathbb{R}_{*}^{2 \times 2}$, as shown by the following lemma.

Lemma 4. A given bivariate VAR model with its coefficient matrix $A \in$ $\mathbb{R}^{2 \times 2}$ is stable, if and only if

$$
|\operatorname{tr}(A)|-1<\operatorname{det}(A)<1 .
$$

Proof. Let $\lambda$ be an eigenvalue of the coeffient matrix $A$. Then such an eigenvalue satisfies

$$
f(\lambda)=\left|A-\lambda I_{2}\right|=\lambda^{2}-\operatorname{tr}(A) \lambda+\operatorname{det}(A)=0 .
$$

If a VAR is stable, this eigenvalue needs to satisfy $|\lambda|<1$. As (24) is rewritten by

$$
f(\lambda)=\left(\lambda-\frac{1}{2} \operatorname{tr}(A)\right)^{2}-\frac{1}{4}\left(\operatorname{tr}(A)^{2}-4 \operatorname{det}(A)\right),
$$

we analyze this condition on (24) for the following two cases with lambda being real or non-real:

1. If $\lambda$ is real, this stability condition is equivalent with

$$
\operatorname{tr}(A)^{2} \leq 4 \operatorname{det}(A), f(1)>0, f(-1)>0,|\operatorname{tr}(A)|<2 .
$$

2. If $\lambda$ is not real, this stability condition is equivalent with

$$
\operatorname{tr}(A)^{2}<4 \operatorname{det}(A),|\lambda|^{2}<1 .
$$


If lambda of (24) is non-real (Case 2.), $\lambda$ (and its conjugate) is

$$
\lambda=\frac{1}{2} \operatorname{tr}(A) \pm \frac{j}{2} \sqrt{\left|\operatorname{tr}(A)^{2}-4 \operatorname{det}(A)\right|},
$$

with imaginary unit denoted by $j$.

With the inequality (27), the stability condition in this case is

$$
\left(\frac{\operatorname{tr}(A)}{2}\right)^{2}<|\lambda|^{2}=\operatorname{det}(A)<1 .
$$

If $\lambda$ of $(24)$ is real, $\operatorname{tr}(A)^{2}-4 \operatorname{det}(A) \geq 0$ and

$$
\begin{aligned}
f(1) & =1-\operatorname{tr}(A)+\operatorname{det}(A)>0 \\
f(-1) & =1+\operatorname{tr}(A)+\operatorname{det}(A)>0 \\
|\operatorname{tr}(A)| & <2 .
\end{aligned}
$$

Combining (30) and (31), we have $|\operatorname{tr}(A)|-1<\operatorname{det}(A)$. This inequality with $(26)$,

$$
C_{0}<\operatorname{det}(A) \leq\left(\frac{\operatorname{tr}(A)}{2}\right)^{2}<C_{1},
$$

where

$$
\begin{aligned}
C_{0} & :=|\operatorname{tr}(A)|-1, \\
C_{1} & :=\min \left(1,\left(\frac{1+\operatorname{det}(A)}{2}\right)^{2}\right) .
\end{aligned}
$$

Find for an arbitrary $A \in \mathbb{R}^{2 \times 2}$ we have the following two inequalities

$$
|\operatorname{tr}(A)|-1 \leq\left(\frac{1}{2} \operatorname{tr}(A)\right)^{2}
$$

and

$$
\operatorname{det}(A) \leq\left(\frac{1+\operatorname{det}(A)}{2}\right)^{2} .
$$

The inequality (34) holds equality for and only for $\operatorname{tr}(A)=2$, and the inequality (34) holds equality for and only for $\operatorname{det}(A)=1$. As both of these equality conditions do not hold under (29), (29) is equivalent with

$$
C_{0}<\left(\frac{\operatorname{tr}(A)}{2}\right)^{2}<\operatorname{det}(A)<C_{1}
$$


Integrating the two inequalities (33) for real $\lambda$ and (36) for non-real $\lambda$, the VAR with the coefficient matrix $A$ is stable, if

$$
C_{0}<\operatorname{det}(A)<C_{1}
$$

and

$$
C_{0}<\left(\frac{\operatorname{tr}(A)}{2}\right)^{2}<C_{1} .
$$

As the inequality $(37)$ implies $0<\frac{1+\operatorname{det}(A)}{2}<1$ and $\operatorname{tr}(A)<2$, (38) is equivalent with

$$
\left(\frac{\operatorname{tr}(A)}{2}\right)^{2}<\left(\frac{1+\operatorname{det}(A)}{2}\right)^{2} .
$$

As the upper bound for $\operatorname{det}(A)$ in $(37)$ can be implied by $\operatorname{det}(A)<1$, it is equivalent with

$$
|\operatorname{tr}(A)|-1<\operatorname{det}(A)<1 .
$$

Thus, the pair of the inequalities (37) and (38) for $A$ is equivalent with the single inequality (40) for $A$.

\subsection{Stability and existence of the solution for the Lyapunov equation}

Intuitively, it would be reasonable that there is a stationary covariance matrix $\hat{\Sigma} \in \mathbb{R}_{+}^{2 \times 2}$ satisfying the Lyapunov equation (4), if the coefficient matrix is $A \in \mathbb{R}_{*}^{2 \times 2}$. However, this is not too trivial, as the opposite may not be always true: The existence of $\hat{\Sigma} \in \mathbb{R}_{+}^{2 \times 2}$ does not imply $A \in \mathbb{R}_{*}^{2 \times 2}$. This relationship between $A$ and $\hat{\Sigma}$ is stated by the following Theorem 1 .

Theorem 1. There is a stationary covariance matrix $\hat{\Sigma} \in \mathbb{R}_{+}^{2 \times 2}$ satisfying the Lyapunov equation (4), if the coefficient matrix is $A \in \mathbb{R}_{*}^{2 \times 2}$. However, the existence of $\hat{\Sigma} \in \mathbb{R}_{+}^{2 \times 2}$ does not imply $A \in \mathbb{R}_{*}^{2 \times 2}$.

Proof. By Lemma 4 and 5 , det $\left(\left(I_{4}-A \otimes A\right)\right)>0$. Thus Lyapunov equation 5 has the solution for $\hat{\Sigma}$, as the matrix $\left(I_{4}-A \otimes A\right)$ is invertible. The converse of this theorem does not hold, as we construct a counter-example of the coefficient matrix $A$ such that $\operatorname{det}\left(\left(I_{4}-A \otimes A\right)\right)<0$, with which there is a $\hat{\Sigma} \in \mathbb{R}_{+}^{2 \times 2}$, but such a VAR is not stable.

In the proof of Theorem 1, we use the following lemma. 
Lemma 5. For an arbitrary VAR model with a base covariance matrix $\Sigma \in$ $\mathbb{R}_{+}^{2 \times 2}$, a cofficient matrix $A \in \mathbb{R}^{2 \times 2}$, and stationary covariance matrix $\hat{\Sigma} \in$ $\mathbb{R}_{+}^{2 \times 2}$, there is the identity:

$$
\operatorname{det}\left(\left(I_{4}-A \otimes A\right)\right)=(1-\operatorname{det}(A))^{2}\left\{(1+\operatorname{det}(A))^{2}-\operatorname{tr}(A)^{2}\right\} .
$$

Proof. Expand the determinant as follows:

$$
\begin{aligned}
\operatorname{det}\left(\left(I_{4}-A \otimes A\right)\right) & =\operatorname{det}\left(\left\{\left(I_{2}-a_{0,0} A\right)\left(I_{2}-a_{1,1} A\right)-a_{0,1} a_{1,0} A^{2}\right\}\right) \\
& =\operatorname{det}\left(\left(I_{2}-A \operatorname{tr}(A)+A^{2} \operatorname{det}(A)\right)\right) . \\
& =(1-\operatorname{det}(A))^{2}\left(\left(1-a_{0,1}-a_{0,0}^{2}\right)\left(1-a_{0,1}-a_{1,1}^{2}\right)-a_{0,1} a_{1,0} \operatorname{tr}(A 4) 4\right) \\
& =(1-\operatorname{det}(A))^{2}\left\{(1+\operatorname{det}(A))^{2}-\operatorname{tr}(A)^{2}\right\} .
\end{aligned}
$$

\section{Design of bivariate timeseries given GCs}

The goal of this manuscript is to derive a design principle of bivariate timeseries generated by a VAR model. The set of parameters in VAR include

- coefficient matrix $A$,

- the base covariance matrix $\Sigma$,

- stationary covariance matrix $\hat{\Sigma}$,

- and two ways of Granger causality $G_{0}, G_{1}$.

There are equality constraints on these variables:

- The variables $A, \Sigma, \hat{\Sigma}$ needs to satisfy the Lyapunov equation (4).

- Granger causality $G_{i}$ is a function of $a_{i, 1-i}, \sigma_{i, i}$, and $\hat{\Sigma}$ (Lemma 2).

Besides, it is important to know the feasibility of a set of parameters in VAR, which constrains the range of these variables:

- the stability: $A \in \mathbb{R}_{*}^{2 \times 2}$ (Section 4 ),

- properness: $\Sigma, \hat{\Sigma} \in \mathbb{R}_{+}^{2 \times 2}$ (Section 4) and $\sigma_{i, i} \leq \hat{\sigma}_{i, i}$ due to the existence of a solution for the Lyapunov equation,

- and the bound for each Granger causality: $G_{i} \in\left[0, \log \gamma_{i}\right]$ (Lemma 3). 
The Lyapunov equation (4) on the matrices can be decomposed into the three equations on the scalar variables as follows. For a coefficient matrix $A=\left(\begin{array}{ll}a_{0,0} & a_{0,1} \\ a_{1,0} & a_{1,1}\end{array}\right)$, let us define two vectors by

$$
a_{0}:=\left(\begin{array}{c}
a_{0,0} \\
a_{0,1}
\end{array}\right), a_{1}:=\left(\begin{array}{c}
a_{1,0} \\
a_{1,1}
\end{array}\right)
$$

Then Lyapunov equation is equivalently written with these vectors $a_{0}, a_{1}$ by

$$
\begin{aligned}
\hat{\sigma}_{0,0}-\sigma_{0,0} & =a_{0}^{\top} \hat{\Sigma} a_{0} \\
\hat{\sigma}_{1,1}-\sigma_{1,1} & =a_{1}^{\top} \hat{\Sigma} a_{1} \\
\hat{\sigma}_{0,1}-\sigma_{0,1} & =a_{0}^{\top} \hat{\Sigma} a_{1} .
\end{aligned}
$$

Equation (46) and (47) in the above implies that each of the vectors $a_{0}$ and $a_{1}$ is on an ellipsis on their plane. This gives the lower bound for $\hat{\sigma}_{i, i} \geq \sigma_{i, i}$ (i.e., one condition of the properness above), as $x^{\top} \hat{\Sigma} x \geq 0$ for any $x \in \mathbb{R}^{2}$ with a positive definite matrix $\hat{\Sigma}$.

By fixing $G_{0}$ and $G_{1}$, it imposes each of the two vectors $a_{0}$ and $a_{1}$ on the two parallel lines by

$$
\left(a_{i}^{\top} \hat{\sigma}_{i}\right)^{2}=\tau_{i}^{2},
$$

where

$$
\hat{\sigma}_{i}:=\left(\hat{\sigma}_{i, 0}, \hat{\sigma}_{i, 1}\right)^{\top}, \tau_{i}^{2}:=\hat{\sigma}_{i, i}^{2}\left(1-\gamma_{i}^{-1} e^{G_{i}}\right) .
$$

Thus, the solution of $a_{i}$ to satisfies the Lyapunov equation and the fixed Granger causality is the four intersections of the ellipsis and the two parallel lines (Figure 1). This ellipsis is obtained by scaling and shearing transformation to the standard circle $a_{0,0}^{2}+a_{0,1}^{2}=1$. This observation gives the angular parametrization of the solution vector $\left(a_{0,0}, a_{0,1}\right)^{\top}$, which is stated by Lemma 6 in the next section.

\subsection{Solution}

In what follows, we start with derivation of the coeffient matrix $A$ as a root of the equality constraint by the Lyapunov equation (4) and the Granger causality, for a fixed proper $\hat{\Sigma}, \sigma_{i, i}$ and $G_{i}$ for each $i=0,1$. The following Lemma 6 gives a necessary condition for the coefficient matrix $A \in \mathbb{R}^{2 \times 2}$ satisfying the equality conditions above. Note that, however, a solution $A$ of this equation does not guarantee $A \in \mathbb{R}_{*}^{2 \times 2}$. 


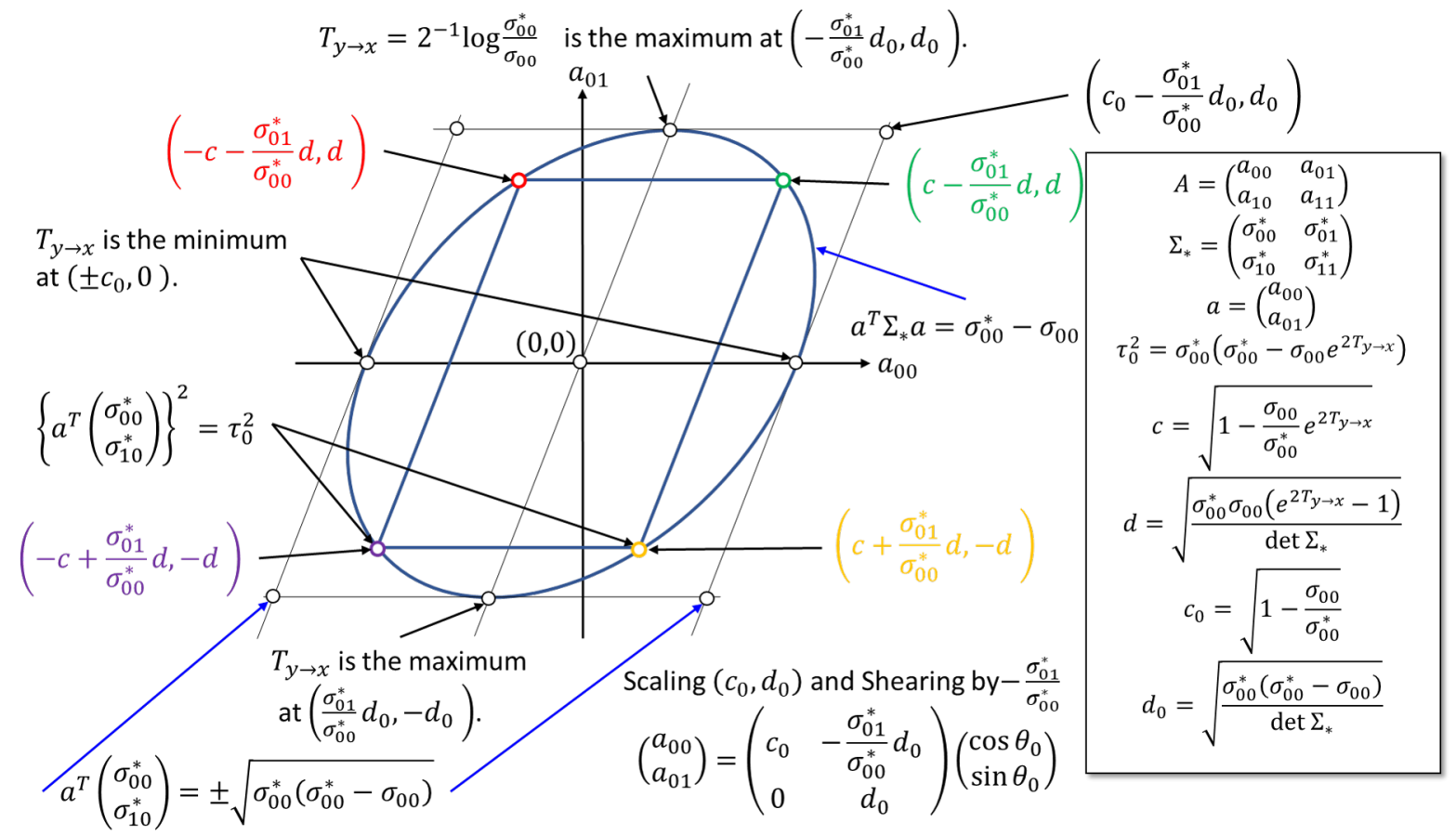

Figure 1: The ellipsis (46) and two parallel lines (49) on the plane $\left(a_{0,0}, a_{0,1}\right) \in \mathbb{R}^{2}$. The solution $\left(a_{0}, a_{1}\right)$ is four intersections of these two. Granger causality takes its maximum with the largest $\left|a_{0,1}\right|$ and its minimum with $\left|a_{0,1}\right|=0$. 
Lemma 6. For a given set of parameters, a positive definite matrix $\hat{\Sigma}=$ $\left(\begin{array}{ll}\hat{\sigma}_{0,0} & \hat{\sigma}_{0,1} \\ \hat{\sigma}_{1,0} & \hat{\sigma}_{1,1}\end{array}\right) \in \mathbb{R}_{+}^{2 \times 2}, \sigma_{i, i} \in\left(0, \hat{\sigma}_{i, i}\right), G_{i} \in\left[0, \log \gamma_{i}\right]$ for each $i=0,1$, suppose that a coefficient matrix $A=\left(\begin{array}{cc}a_{0,0} & a_{0,1} \\ a_{1,0} & a_{1,1}\end{array}\right) \in \mathbb{R}^{2 \times 2}$, satisfies the set of the equations

$$
\left\{\begin{array}{l}
a_{0}^{\top} \hat{\Sigma} a_{0}=\hat{\sigma}_{0,0}-\sigma_{0,0} \\
a_{1}^{\top} \hat{\Sigma} a_{1}=\hat{\sigma}_{1,1}-\sigma_{1,1} \\
\left(\hat{\sigma}_{0}^{\top} a_{0}\right)^{2}=\tau_{0}^{2} \\
\left(\hat{\sigma}_{1}^{\top} a_{1}\right)^{2}=\tau_{1}^{2}
\end{array},\right.
$$

where for $i=0,1$

$$
\hat{\sigma}_{i}:=\left(\hat{\sigma}_{i, 0}, \hat{\sigma}_{i, 1}\right)^{\top}, \tau_{i}^{2}:=\hat{\sigma}_{i, i}^{2}\left(1-\gamma_{i}^{-1} e^{G_{i}}\right) .
$$

Any coefficient matrix $A$ of a root of this equation (50) is in the form of

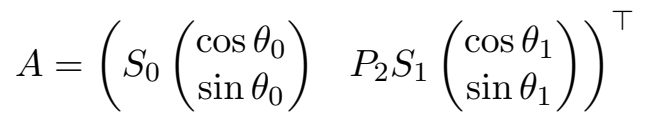

where each pair of the angles $\theta_{0} \in[0,2 \pi)$ and $\theta_{1} \in[0,2 \pi)$ takes one of the two or four pairs satisfying for each $i=0,1$

$$
\sin ^{2} \theta_{i}=\frac{e^{G_{i}}-1}{\gamma_{i}-1}
$$

and

$$
P_{2}:=\left(\begin{array}{ll}
0 & 1 \\
1 & 0
\end{array}\right), S_{i}:=\sqrt{1-\gamma_{i}^{-1}}\left(\begin{array}{cc}
1 & -\frac{\hat{\sigma}_{i, 1-i}}{\hat{\sigma}_{i, i}} \\
0 & 1
\end{array}\right)\left(\begin{array}{cc}
1 & 0 \\
0 & \frac{\hat{\sigma}_{i, i}}{\sqrt{\operatorname{det}(\hat{\Sigma})}}
\end{array}\right)
$$

Proof. Find that the following pair of equations in the (50) is symmetric under exchange of $i=0,1$ :

$$
\left\{\begin{array}{l}
a_{i}^{\top} \hat{\Sigma} a_{i}=\hat{\sigma}_{i, i}-\sigma_{i, i} \\
\left(\hat{\sigma}_{i}^{\top} a_{i}\right)^{2}=\tau_{i}^{2}
\end{array} .\right.
$$

Thus, we solve this for $i=0$ below, and it holds for $i=1$.

Solving the second equation of (53) for $a_{0,0}$, we have

$$
a_{0,0}=\frac{ \pm \tau_{0}-a_{0,1} \hat{\sigma}_{0,1}}{\hat{\sigma}_{0,0}}
$$


Inserting this to the first equation of (53), we have

$$
a_{0,1}^{2}=\frac{\hat{\sigma}_{0,0}^{2}\left(1-\gamma_{0}^{-1}\right)-\tau_{0}^{2}}{\operatorname{det}(\hat{\Sigma})} .
$$

Inserting $\tau_{0}^{2}=\hat{\sigma}_{0,0}^{2}\left(1-\gamma_{0}^{-1} e^{G_{0}}\right)$,

$$
a_{0,1}= \pm \sqrt{\frac{\hat{\sigma}_{0,0} \sigma_{0,0}\left(e^{G_{0}}-1\right)}{\operatorname{det}(\hat{\Sigma})}}
$$

Inserting this to (54), we have at most four vectors $a_{0}=\left(a_{0,0}, a_{0,1}\right)^{\top}$ as the solution of (53) for $i=1$ :

$$
a_{0}=\left(\begin{array}{c}
c_{0}-\frac{\hat{\sigma}_{0,1}}{\hat{\sigma}_{0,0}} d_{0} \\
d_{0}
\end{array}\right),\left(\begin{array}{c}
c_{0}+\frac{\hat{\sigma}_{0,1}}{\hat{\sigma}_{0,0}} d_{0} \\
-d_{0}
\end{array}\right),\left(\begin{array}{c}
-c_{0}-\frac{\hat{\sigma}_{0,1}}{\hat{\sigma}_{0,0}} d_{0} \\
d_{0}
\end{array}\right),\left(\begin{array}{c}
-c_{0}+\frac{\hat{\sigma}_{0,1}}{\hat{\sigma}_{0,0}} d_{0} \\
-d_{0}
\end{array}\right),
$$

where for $i=0,1$

$$
c_{i}:=\sqrt{1-\gamma_{i}^{-1} e^{G_{i}}}, d_{i}:=\sqrt{\frac{\hat{\sigma}_{i, i} \sigma_{i, i}\left(e^{G_{i}}-1\right)}{\operatorname{det}(\hat{\Sigma})}} .
$$

By symmetry to $i=0,1$, there are at most four vectors as the solution of (53) for $i=1$ :

$P_{2} a_{1}=\left(\begin{array}{c}a_{1,1} \\ a_{1,0}\end{array}\right)=\left(\begin{array}{c}c_{1}-\frac{\hat{\sigma}_{1,0}}{\hat{\sigma}_{1,1}} d_{1} \\ d_{1}\end{array}\right),\left(\begin{array}{c}c_{1}+\frac{\hat{\sigma}_{1,0}}{\hat{\sigma}_{1,1}} d_{1} \\ -d_{1}\end{array}\right),\left(\begin{array}{c}-c_{1}-\frac{\hat{\sigma}_{1,0}}{\hat{\sigma}_{1,1}} d_{1} \\ d_{1}\end{array}\right),\left(\begin{array}{c}-c_{1}+\frac{\hat{\sigma}_{1,0}}{\hat{\sigma}_{1,1}} d_{1} \\ -d_{1} .\end{array}\right)$

Find that these four solution vectors parameterized by

$$
a_{0}=S_{0}\left(\begin{array}{c}
\cos \theta_{0} \\
\sin \theta_{0}
\end{array}\right), a_{1}=P_{2} S_{1}\left(\begin{array}{c}
\cos \theta_{1} \\
\sin \theta_{1}
\end{array}\right),
$$

satisfy (53), if $\left(\theta_{0}, \theta_{1}\right)$ holds (52), with the trigonometric identity $\cos ^{2} \theta_{i}+$ $\sin ^{2} \theta_{i}=1$ and

$$
S_{0}^{\top} \hat{\Sigma} S_{0}=\left(\hat{\sigma}_{0,0}-\sigma_{0,0}\right) I_{2}, S_{1}^{\top} P_{2}^{\top} \hat{\Sigma} P_{2} S_{1}=\left(\hat{\sigma}_{1,1}-\sigma_{1,1}\right) I_{2} .
$$




\subsection{Sufficiency of the solution}

For a given set of the parameters, Lemma 6 in the previous section gives a set of solutions of the coefficient matrix $A$ for the Lyapunov equation. Note that not all of these solutions $A$ is feasible, in the sense that it satisfies all constraints such as stability of $A$ and properness of $\Sigma$. The following lemmas provide the sufficient condition for a solution $A$ by checking properness of $\Sigma$ and stability of $A$.

Lemma 7. Suppose $A$ is a solution of the equation (50) in Lemma 6, represented by a pair of $\left(\theta_{0}, \theta_{1}\right)$. In this case, $\Sigma \in \mathbb{R}_{+}^{2 \times 2}$, if and only if

$$
\cos \hat{\eta}-\left(\gamma_{0} \gamma_{1}\right)^{-\frac{1}{2}} \leq \hat{\gamma}_{0} \hat{\gamma}_{1} \cos \left(\hat{\eta}-\theta_{0}-\theta_{1}\right) \leq \cos \hat{\eta}+\left(\gamma_{0} \gamma_{1}\right)^{-\frac{1}{2}}
$$

where $\hat{\eta} \in[0,2 \pi]$ is the angler parameterization of the correlation coefficient defined by $\cos \hat{\eta}:=\frac{\hat{\sigma}_{0,1}}{\hat{\sigma}_{0,0} \hat{\sigma}_{1,1}}$ and $\hat{\gamma}_{i}=\sqrt{1-\gamma_{i}^{-1}}$.

Proof. Applying the polar representation of the $a_{0}, a_{1}$ in (51) in Lemma 6 to the third equation (48) of the Lyapunov equation, we have

$$
\hat{\sigma}_{0,1}-\sigma_{0,1}=\sqrt{\left(\hat{\sigma}_{0,0}-\sigma_{0,0}\right)\left(\hat{\sigma}_{1,1}-\sigma_{1,1}\right)} \cos \left(\hat{\eta}-\theta_{0}-\theta_{1}\right) .
$$

By the positive definiteness of $\Sigma, \sigma_{0,1}^{2} \leq \sigma_{0,0} \sigma_{1,1}$. This inequality applied to (61) gives the lemma.

If we have

$$
\hat{\gamma}_{0} \hat{\gamma}_{1} \leq \cos \hat{\eta}+\left(\gamma_{0} \gamma_{1}\right)^{-\frac{1}{2}} \wedge \cos \hat{\eta}-\left(\gamma_{0} \gamma_{1}\right)^{-\frac{1}{2}} \leq-\hat{\gamma}_{0} \hat{\gamma}_{1},
$$

(60) holds for any pair of angles $\left(\theta_{0}, \theta_{1}\right)$. This condition is

$$
|\cos \hat{\eta}| \leq\left(\gamma_{0} \gamma_{1}\right)^{-\frac{1}{2}}-\hat{\gamma}_{0} \hat{\gamma}_{1}
$$

or

$$
\left|\hat{\sigma}_{0,1}\right| \leq \sqrt{\sigma_{0,0} \sigma_{1,1}}-\sqrt{\left(\hat{\sigma}_{0,0}-\sigma_{0,0}\right)\left(\hat{\sigma}_{1,1}-\sigma_{1,1}\right)} .
$$

By Lemma 4 , a stable VAR has $A$ satisfying

$$
-1+|\operatorname{tr}(A)|<\operatorname{det}(A)<1
$$

Using the angular notation of the solution $A$ with $\theta_{0}, \theta_{1}$, we have

$$
\operatorname{det}(A)=\hat{\gamma}_{0} \hat{\gamma}_{1} \frac{\sin \left(\hat{\eta}-\theta_{0}-\theta_{1}\right)}{\sin \hat{\eta}}
$$


and

$$
\operatorname{tr}(A)=\frac{\hat{\gamma}_{0} \sin \left(\hat{\eta}-\theta_{0}\right)+\hat{\gamma}_{1} \sin \left(\hat{\eta}-\theta_{1}\right)}{\sin \hat{\eta}} .
$$

Thus, inserting these to the stability condition (23), we have

$$
-\sin \hat{\eta}+\left|\hat{\gamma}_{0} \sin \left(\hat{\eta}-\theta_{0}\right)+\hat{\gamma}_{1} \sin \left(\hat{\eta}-\theta_{1}\right)\right|<\hat{\gamma}_{0} \hat{\gamma}_{1} \sin \left(\hat{\eta}-\theta_{0}-\theta_{1}\right)<\sin \hat{\eta} .
$$

\section{Summary and remarks}

In this manuscript, we analyzed bivariate VAR model, and explore the relationship between the parameters in VAR and its pair of Granger causality. Granger causality $G_{0}, G_{1}$ (in the form of angular $\theta_{0}, \theta_{1}$ ) is independently from the stationary covariance $\hat{\sigma}_{0,1}$ of the bivariate timeseries up to the constrant Lemma 7 . Or the stationary covariance and variance of $\hat{\Sigma}$ and $\Sigma$ needs to be constrained by (62), in order to let Granger causality be any value $G_{i} \in\left[0, \log \gamma_{i}\right]$. Thus, our analysis revealed the trade-off like relationship of the stationary covariance and Granger causality.

This half-constraint relationship - Granger causality is almost free from covariance of the bivariate timeseries, but needs to satisfy the range constraint imposed by covariance matrix. Perhaps, this loose logical connection between Granger causality and covariance would cause a conceptual confusion of temporal inter-relationship between two moving objects in literature. In literature, a sort of terms such as "synchronization", "contingency", "interaction", and "lead-follow relationship" has been used to express some temporal inter-relationship between motions. Some of these terms was intended to indicate covariance, and the other was intended to indicate Granger causality, or a mixture of them.

Even if animacy perception is related to Granger causality but not to covariance, a carefully designed experiment needs to be conducted to clarify this conceptual confusion. Although covariance and Granger causality have the mutually constraining relationship as analyzed in this manuscript, Granger causality can be manipulable to a large extent with a fixed covariance value. Thus, we can discriminate the effect of Granger causality from the effect of covariance on animacy perception by carefully designing the psychological experiment. 


\section{Acknowledgements}

This work is supported by JSPS KAKENHI Grant Number JP $20 \mathrm{H04994}$ and JST, PRESTO Grant Number JPMJPR20C9, Japan.

\section{References}

[1] Lionel Barnett, Adam B. Barrett, and Anil K. Seth. Granger causality and transfer entropy are equivalent for gaussian variables. Physical Review Letters, 103(23):238701-1-4, 2009.

[2] John N. Bassili. Temporal and spatial contingencies in the perception of social events. Journal of Personality and Social Psychology, 33(6):680$685,1976$.

[3] Winand H. Dittrich and Stephen E.G. Lea. Visual perception of intentional motion. Perception, 23(3):253-268, 1994.

[4] Clive W.J. Granger. Investigating causal relations by econometric models and cross-spectral methods. Econometrica, 37(3):424-438, 1969.

[5] Fritz Heider and Marianne Simmel. An experimental study of apparent behavior. The American Journal of Psychology, 57(2):243-259, 1944.

[6] Brian J. Scholl and Patrice D. Tremoulet. Perceptual causality and animacy. Trends in Cognitive Sciences, 4(8):299-308, 2000.

[7] Kohsuke Takahashi and Katsumi Watanabe. Synchronous motion modulates animacy perception. Journal of Vision, 15(8):1-17, 2015.

[8] Patrice D. Tremoulet and Jacob Feldman. Perception of animacy from the motion of a single object. Perception, 29(8):943-951, 2000. 